excluded. Finally 88 patients $(59$ females, $67.1 \%$ and 29 males, 32.9\%) aged 30 to 83 years (mean: $52 \pm 13$ years) with 97 aneurysms (57 ruptured, 58.7\% and 40 unruptured, 41.3\%) were included. Aneurysm locations were internal carotid artery (36 aneurysms, 30.9\%), anterior communicating and anterior cerebral arteries (31 aneurysms, 31.9\%), middle cerebral artery (16 aneurysms, 16.5\%), vertebrobasilar system (14 aneurysms, 14.4\%). Seventy-seven aneurysms (79.4\%) were treated with simple coiling and 20 (20.6\%) with balloonassisted coiling.

Aneurysm coiling was feasible in all aneurysms. Thromboembolic events with or without clinical worsening were encountered in $14 / 97$ procedures (14.4\%) intraoperative rupture in 6 aneurysms (6.6\%), all without clinical worsening. Overall the procedural morbidity and mortality were encountered in 2 patients $(2.1 \%)$ and 1 patient (1.0\%), respectively.

Post-operative anatomical results were complete aneurysm occlusion in 79 aneurysms (81.4\%), neck remnant in 13 aneurysms (13.4\%) and aneurysm remnant in 5 aneurysms (5.2\%).

Conclusion Preliminary results in this prospective series show good safety and efficacy of Barricade coils in aneurysm treatment.

Disclosures L. Pierot: 2; C; Blockade. M. Zidan: None. C. Foussier: None. G. Metaxas: None. S. Soize: None.

\section{E-042 EFFICACY OF AN AVM CLASSIFICATION SYSTEM THAT DIRECTS ENDOVASCULAR THERAPIES ACCURATELY}

W Yakes. Vascular Malformation Center, Englewood, CO

10.1136/neurintsurg-2016-012589.114

Purpose To determine if AVM angioarchitecture characteristics can be predictive and direct specific curative endovascular procedures accurately and consistently to treat high-flow malformations.

Materials and methods Angiographic analysis of high-flow vascular malformations determined 4 major angioarchitectures. Type I: Direct arterial/arteriolar to vein/venule connection; e. g., as commonly seen in pulmonary AVF, congenital renal AVF, etc. Type II: Arterial/arteriolar connections to a "nidus" that then have several out-flow veins with no intervening capillary beds in any of the vascular interconnections. Type
IIIa: Arterial/arteriolar connections to an aneurysmal vein ("nidus" is the vein wall) that drains into a dominant out-flow vein with no intervening capillary bed in these connections. Type IIIb: Same angioarchitecture as Type IIIa, except that there are more than one (several) out-flow veins. Type IV: "Infiltrative" form of AVM whereby innumerable micro-arteriolar branches fistulize through a tissue (e.g., ear) totally infiltrating it, shunting into multiple out-flow veins. Capillary beds also exist in the tissue and are mixed with the innumerable AVFs. Without the capillaries the tissue could not be viable, therefore must be present.

Results Type I: Can be effectively treated with mechanical devices; e.g., coils, Amplatzer Plugs, etc. Type II: Can be effectively treated with ethanol embolization. Type IIIa: Can be effectively treated by transcatheter ethanol, retrograde vein catheter access or direct puncture access of the aneurysmal vein and treatment with ethanol and coils, or even by coils alone. Type IIIb: Can be effectively treated as above, but can be more challenging by the vein route as more veins (not a single out-flow vein) require closure. Type IV: Can be effectively treated by transcatheter or direct puncture of the innumerable microfistulous AVFs by embolization with 50\%-50\% ethanol non-ionic contrast mixture.

Conclusion This never before reported classification system has a direct impact on determining the curative endovascular and direct puncture embolization procedures and also determines the embolic agents that will successfully treat complex AVMS in the body.

Disclosures W. Yakes: None.

\section{E-043 REVERSIBLE CEREBRAL VASOCONSTRICTION SYNDROME: NON-INVASIVE IMAGING FINDINGS AND ANGIOGRAPHIC EVALUATION}

K de Macedo Rodrigues, R Hito, D Takhtani, J Lozano, A Wakhloo, A Puri. Radiology, University of Massachusetts Medical School, Worcester, MA

\subsection{6/neurintsurg-2016-012589.115}

Purpose Reversible Cerebral Vasoconstriction Syndrome (RCVS) is a transient disorder of cerebral arterial auto regulation that may have a non-specific clinical presentation, most typically initiating as an acute episode of severe (thunderclap) headache. The clinical scenario and the non-invasive imaging

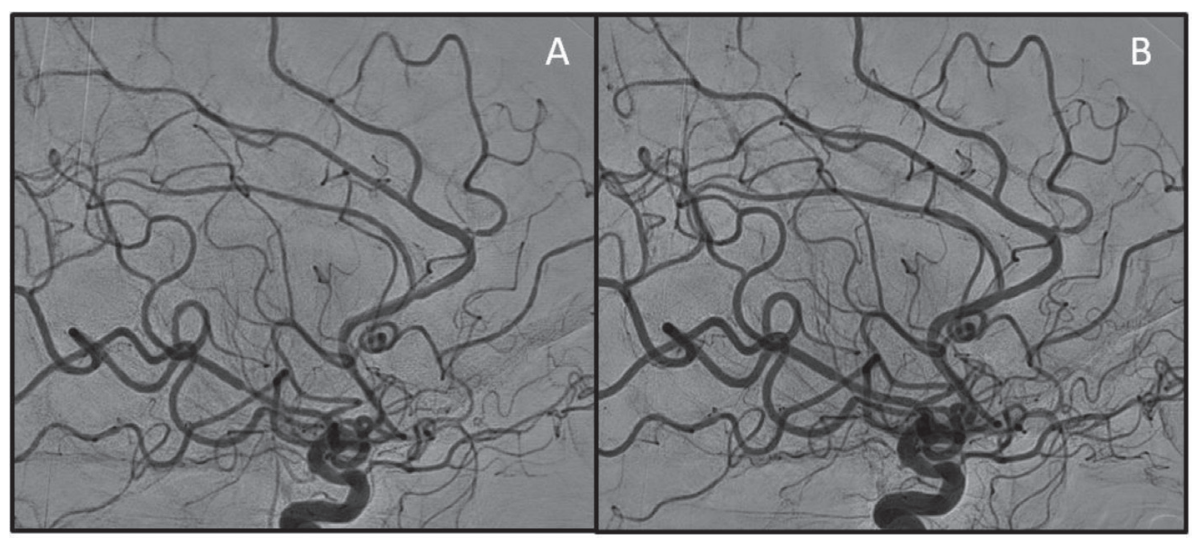

Abstract E-043 Figure 1 48-year old female presenting with severe headache and evidence of subarachnoid hemorrhage on head CT (not shown). No aneurysm or other causes for intracranial bleed was demonstrated on DSA. (A) Multiple segmentel areas of vasoconstrictions of the MCA and ACA branches are demonstrated. (B) There is significant improvement of the degree if vasoconstriction after intra-arterial administration of calcium channel blocker. 
findings are similar to those seen in more common pathologies making it a challenging diagnosis if the entity is not a consideration in the initial evaluation. RCVS presentation and initial imaging findings may mimic a ruptured aneurysm, stroke, migraine and posterior reversible encephalopathy syndrome, among others. Our aim is to describe the entity of RCVS and demonstrate the common and uncommon imaging findings seen on CT, MRI, CT angiography, with emphasis on conventional digital subtraction arteriogram pre and post endovascular treatment.

Approach/methods A multimodality pictorial review of cases from our institution is presented, including non-contrast head CT, CT angiogram, MRI, MR angiogram and conventional digital subtraction arteriogram (DSA). Diagnosis of RCVS was confirmed by imaging demonstration of reversibility of arterial constriction on follow-up imaging or after intra-arterial therapy.

Findings/discussion We present indirect findings on non-invasive imaging, such as transient cerebral edema, convexal subarachnoid hemorrhage, intraparenchymal hemorrhage and cerebral. Direct signs of single or multivessel arterial vasoconstriction are seen on CTA, MRA and confirmed on DSA. Reversibility of the vasoconstriction is demonstrated after intra-arterial administration of calcium channel blocker. Conditions such as diffuse atherosclerotic arterial narrowing, vasculitis, posterior reversible encephalopathy, cerebral edema secondary to venous congestion and vasospasm secondary to aneurysmal subarachnoid hemorrhage were among the pathologies considered prior to a definitive diagnosis of RCVS.

Summary/conclusion Familiarity with the imaging findings of RCVS is of critical importance to direct appropriate and timely clinical management. Atypical subarachnoid hemorrhage, unexplained cerebral edema and hemorrhage, as well as single or multivessel diffuse narrowing with a relatively normal appearing brain should raise suspicion of RCVS. Suspicion can be confirmed by demonstrating the reversibility of vasoconstrictions after intra-arterial administration of calcium channel blocker or at follow-up imaging.

Disclosures K. de Macedo Rodrigues: None. R. Hito: None. D. Takhtani: None. J. Lozano: None. A. Wakhloo: 1; C; NIH, Philips Healthcare, Wyss Institute. 2; C; Codman Neurovascular and Stryker Neurovascular. 4; C; co-founder of InNeuroCo Inc. and major stockholder; stocks in EpiEB and Pulsar Medical.. 6; C; speaker: Harvard Postgraduate Course, Miami Cardiovascular Institute. A. Puri: 1; C; Stryker Neurovascular and Covidien. 2; C; Codman Neurovascular, Stryker Neurovascular and Covidien. 4; C; InNeuroCo Inc. 6; C; speaker: Miami Cardiovascular Institute.

\section{E-044 STENT RETRIEVER THROMBECTOMY AND LOCAL ASPIRATION WITH A NAVIGABLE AND FLEXIBLE SECOND GENERATION SUPPORT CATHETER FOR ACUTE ISCHEMIC STROKE: THE "SOL-ARC" TECHNIQUE}

A Onofrio, A Miller, J Hise, I Thacker, J Haithcock, D Graybeal, K Layton. Radiology, Baylor University Medical Center, Dallas, TX

\subsection{6/neurintsurg-2016-012589.116}

Multiple recent randomized controlled trials have proven the benefit of mechanical thrombectomy using stent retrievers for emergent large vessel occlusion (ELVO). ${ }^{1-5}$ Techniques currently used for endovascular treatment of stroke employ either direct aspiration, stent retriever thrombectomy or a combination of both. When stent retrievers are used, temporary flow arrest with an extracranial balloon guide catheter or assisted local aspiration with a large bore intracranial suction catheter is recommended. This allows for more complete recanalization and prevents embolization to previously uninvolved territories. Recent advancements in large bore intracranial suction catheter technology have made it easier to utilize coaxial stent retriever thrombectomy assisted by local aspiration. At our high-volume comprehensive stroke center, we have found the second generation large bore intracranial Arc support catheter (ev3 Neurovascular, Irvine, CA) to be extremely effective when used in combination with the Solitaire stent retriever (ev3 Neurovascular, Irvine, CA). We have found the Arc support catheter to be much more navigable, less prone to kinking and easier to deliver into the M1 segment (without causing spasm) than the first generation local aspiration catheters. Additionally, the Arc support catheter is less costly than the currently available suction catheters. The Sol-Arc technique begins with placement of a stent retriever device across the embolic occlusion by deployment though a 021 or 027 microcatheter. This microcatheter is placed coaxially through the Arc support catheter which is positioned just proximal to the embolus. After waiting 5 minutes, the stent retriever is pulled inside the Arc support catheter which is simultaneously aspirated. Subsequently, the Arc support catheter is removed while aspirating the guiding sheath in the neck. This technique should allow for faster, safer and more successful stent retriever thrombectomy when used in conjunction with local aspiration.

Disclosures A. Onofrio: None. A. Miller: None. J. Hise: None. I. Thacker: None. J. Haithcock: None. D. Graybeal: None. K. Layton: None.

\section{E-045 PRODUCT COST AND UTILIZATION IN MECHANICAL THROMBECTOMY: A COMPARISON OF TECHNIQUES}

${ }^{1} \mathrm{P}$ Brown, ${ }^{2} \mathrm{M}$ Cobb, ${ }^{1} \mathrm{~T}$ Smith, ${ }^{2} \mathrm{~A}$ Zomorodi, ${ }^{2} \mathrm{~L}$ Gonzalez. ${ }^{1}$ Radiology, Duke University, Durham, NC; ${ }^{2}$ Neurosurgery, Duke University, Durham, NC

\subsection{6/neurintsurg-2016-012589.117}

Introduction/purpose Interventional stroke management has seen a period of explosive growth in the wake of recent trials demonstrating improved outcomes in patients who undergo timely and appropriate procedural management. The two most common interventional techniques involve the use of stent retrieval devices and suction aspiration. While clear clinical benefit to the patient has been seen in the use of these devices, it is not altogether clear the financial implications to the patient and/or his or her insurer for the use of these devices. In a period of heightened sensitivity to healthcare costs, it is reasonable to consider the material cost to the patient and healthcare system for utilization of these new techniques. The goal of our study is to determine the difference, if any, in cost to the healthcare system in performance of interventional thrombectomy by stent retrieval or suction aspiration.

Materials and methods We examined data for interventional stroke management cases performed for M1 segment occlusions at Duke University Medical Center from the period of January 2014 to January 2016. We analyzed the amount billed to the patient for products utilized during these cases. Cases were subdivided into those only utilizing stent retrieval devices (Solitaire $^{\mathrm{TM}}$; Covidien, Dublin, Ireland) and those only 